\title{
E-QUESTION PAPER GENERATION SYSTEM: A REVIEW
}

\author{
Chaiti Sen Gupta, Arijit Kumar Datta, Rimpi Datta, Arnima Das \\ Department of ECE, Narula Institute of Technology, Kolkata, India
}

\begin{abstract}
This paper presents a study of E-QUESTION PAPER GENERATION SYSTEM. As Testing is an important part for Artificial Intelligent based agents, student assessment is also an important part of teaching. In our education system this assessment is done by examination. For this reason Question paper setting has always consistently been a matter of interest. Quality of any question paper is based on various design parameters related to it such as whether the question paper assesses different problem solving skills, whether it cover all modules or chapters from the syllabus and whether it contains various difficulty levels or not. In this paper we are proposing to develop a system that will generate a smart question paper from different sets of question papers using the Artificial Intelligence technology. The smart question paper generated through this system will be secure, fast, streamlined as well as randomized. The process developed in the system will analyze and compose the question paper using an algorithm with efficiency and result into success. The user will input the sets of question papers. Then the system will ask to provide various information for the finalized question paper that it will generate like what will be the marks for each sections, what will be the difficulty level etc. This kind of question paper will be very much helpful in the university where various sets of questions are generated by various professors and a finalized question is needed that will cover all the sets. [2]
\end{abstract}

Keywords: Question Paper, Question Paper Generation, Artificial Intelligent, ELearning.

Cite this Article: Chaiti Sen Gupta, Arijit Kumar Datta, Rimpi Datta, Arnima Das, E-Question Paper Generation System : A Review, International Journal of Electronics and Communication Engineering and Technology, 10(5), 2019, pp. 1-4.

http://iaeme.com/Home/issue/IJECET?Volume=10\&Issue $=5$ 


\section{INTRODUCTION}

Today, education is the most important way of achieving success. Proper examinations help students to improve their quality. Thus we are presenting an Automated Question Paper Generator System that can reduce time consumption by replacing the conventional method of question paper generation system. It also needs lesser man power. We have implemented a role-based hierarchy which restricts access to the users. The system also deploys security mechanisms that prohibit duplication of question papers. Question Paper Generator is special and unique software, which used in school, institutions, colleges, test paper setters who want to have a huge database of questions for frequent generation of question. This software can be implemented in various medical, engineering and coaching institutes for theory paper. Generation of question papers through a question bank is an important activity in learning management systems and educational institutions. The quality of question paper is based on various design constraints such as whether a question paper assesses different problem solving skills, whether it covers all units from the syllabus of a course and whether it covers various difficulty levels.[4] The algorithm is illustrated in the paper by using four constraints namely question paper format, coverage of syllabus, coverage of difficulty levels and coverage of cognitive level. The algorithm presented is extensible to support any number of user defined constraints. Preparing the exam questions is very challenging, tedious and time consuming for the instructors. Thus with the help of this paper we present the solution in form of Automatic Question Paper Generator System (QGS).The design process performs the scrutiny and composes the examination paper using an efficient algorithm with a high rate of success. From the entered input, the examination paper will be generated automatically. The final paper may be stored as 'Word Document' files. The system shows characteristics like simple operation, a great interface, good usability, immense security, and high stability along with reliability. [1]

\section{PROPOSED METHODOLOGY}

A design of suitable automated system for generating question papers and managing related data may prove vital in an Educational Institute. The proposed intelligent examination system consists of four modules such as the user management, course management, question and its answer management, examination and its evaluation management etc. Its architecture is shown in Figure 1. The proposed system is designed by using machine learning based techniques. It also applied logical design to get the suitable intelligent question bank system framework.

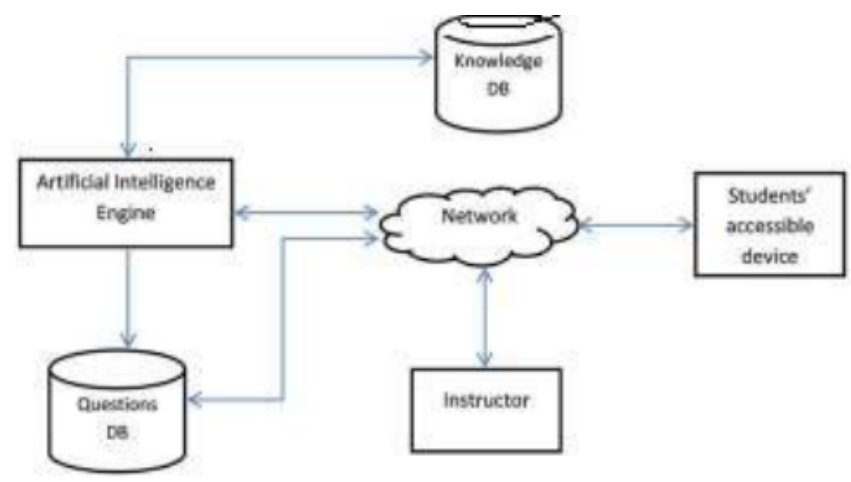

Figure 1 Architecture of Proposed Framework 
In the Question bank system method, the question bank system consists of sets of 100 questions which are created from to the students' syllabuses which is a mixture of medium, easy and hard. Selection of the questions is random generated and intelligence based. The passing percentage of each level of exam is 70. Depending up on the level of marks obtained in each level and in each question, the algorithm will choose the level of questions. The evaluation process is also random generated automated so that the algorithm can find the level of understanding of students in each section of the subject. The main advantage of the proposed method is analyzing the student's understanding level and mastery level in the respective chapters. The proposed methodology will adjust the questions based the students understanding level and mastery level. The question database will have the questions uploaded by instructors. [5]

In Evolution section, an Artificial Intelligence based intelligent system is used for allocating the marks for each questions based on the correct answers. The algorithm is trained with questions and its respective answers. The AI agent is an intelligent module which allocates the correct answers for the appropriate questions and it also allocates the set of questions to a specific student based on the register number so that the question sets cannot be repeated among the others. The AI based subsystem can generate the questions and send them to the device where the student can access the question and he can write the answers The proposed system of an intelligent question bank and examination system consists of four parts: 1. User Management; 2. Course Management; 3. Question and its answer Management; 4. Examination and its evaluation Management. The role of user management is to monitor the functions such as login, editing the profile and allowing permission for the users for accessing the system. Login function monitors the users who were accessing the system. It also maintains the correct username, password and sign up function before login to the system. Also, the Users can edit their profiles, by changing the password, email and their profile picture etc. It manages the setting permission to different types of users such as the student, teacher or administrator. Question and its answer management is to monitor the questions and its corresponding answers by adding, deleting and updating the questions and its corresponding answers, setting the questions, assigning the answers. Artificial Intelligence based methodology for grouping the questions and its answers automatically based on the register numbers of the students. [4]

\section{FUTURE SCOPE OF THE SYSTEM}

Free from tension of collecting question as Built in ready to use question bank is available. One can make sets of paper from one database. This software assures no duplicity of questions in database. Question paper once generated can be downloaded or emailed to the admin who has logged in. this software provides well-arranged syllabus of related subjects in chapters or modules. Three level of difficulty of questions are provided i.e. Easy, Medium, and Difficult. Question paper is generated with great ease and accuracy in less than a minute. Keeps record of generated question paper. Question paper can be generated and viewed only by admin and password security is provided. Fully customization of test paper institute name, time, instructions and maximum marks. This software provides unique user authentication facilities. Database backup and restore backup facilities are also provided. [3] 


\section{CONCLUSION}

Generation of question papers through a question bank is an important activity in learning management systems and educational institutions. The quality of question paper is based on various design constraints such as whether a question paper assesses different problem solving skills, whether it covers all units from the syllabus of a course and whether it covers various difficulty levels. The algorithm is illustrated in the paper by using four constraints namely question paper format, coverage of syllabus, coverage of difficulty levels and coverage of cognitive level. This is a web-Based System. Our future effort is to employee different types of randomization as well as addition to Question Generation, we can enhance the same software by making provision to produce question from online test. Therefore, the resultant automated system for Question Paper Generation provides improvement in terms of controlled access to the resources, random generation of question papers and a secure platform.

\section{REFERENCES}

[1] Cong Y. Functional design of English online examination system based on ASP technology. Proc $3^{\text {rd }}$ International Conference on Education, Management, Arts, Economics and Social Science; 2016. p. 1140-4.

[2] G-Asks: An Intelligent Automatic Question Generation System for Academic Writing Support by Ming Liu and Rafael A. Calvo.

[3] Microsoft Developer Network (MSDN): http://msdn2.microsoft.com/enus/default.aspx: This is a valuable online resource, and is a must for any developer using Microsoft tools.

[4] Automatic Question Generation using Discourse Cues by Manish Agarwal, Rakshit Shah_and Prashanth Mannem

[5] Automatic Cloze-Questions Generation by Annamaneni Narendra, Manish Agarwal and Rakshit shah LTRC, IIIT-Hyderabad, India. 